\title{
correspondence
}

\section{Addiction as a brain disease does not promote injustice}

To the Editor - The recent World View by Carl Hart, 'Viewing addiction as a brain disease promotes social injustice ${ }^{\text {, }}$, raises important questions. Is addiction a brain disease? Does this view of addiction promote discrimination?

Drug use, as the author notes, does not become problematic in most people. Conversely, addiction, or substance use disorder (SUD), is defined as a disease by the American Psychiatric Association ${ }^{2}$.

But is it a brain disease? Hart notes that "there are virtually no data in humans indicating that addiction is a disease of the brain, in the way that Huntington's or Parkinson's are diseases of the brain. With these illnesses one can look at the brain of affected individuals and make accurate predictions about the disease involved and their symptoms." From this, criteria for a 'brain disease' can be inferred: (1) the condition manifests in the brain in an observable way; and (2) brain observations can be used to make accurate predictions about the disease (presumably progression) and the individual's symptoms. However, neuroimaging studies have shown that neurobiological function in SUDs differs markedly from healthy individuals, and these differences can have comparable effect sizes to those in diseases like Huntington's or Parkinson's ${ }^{3-7}$. Additionally, neither structural nor functional neuroimaging allows accurate predictions about individuals' disease progression or symptoms of these and many other neurological diseases: prognosis and management is based on clinical criteria. Similarly, structural/functional neuroimaging cannot facilitate accurate predictions in individuals with primary psychiatric disorders. Thus, according to Hart's criteria, SUD is not a brain disease, but neither are other psychiatric conditions (for example, schizophrenia) or many neurological disorders.

If addiction is a brain disease, does this eliminate the role of psychosocial factors? Hart sets up a dichotomy between the social and the biological as mutually exclusive. Yet, as inherently social creatures, the social is biological. Stress, trauma, poverty, and socioeconomic status are 'written' on the brain in the complex interplay between individuals, their environment, and their experiences $^{8,9}$. Identifying social and/ or behavioural factors contributing to a disease is often key to treating that disease. Does the impact of diet, exercise, and social status undermine the recognition that cardiovascular disease is a disease? Similarly, the influence of discrimination and poverty on the development of SUD is not inconsistent with its status as a disease manifest in the brain. These psychosocial determinants have not been researched comprehensively, but the psychosocial and neurobiological are not mutually exclusive.

Finally, does conceptualizing SUDs as brain disorders drive manifestly discriminatory drug policies? To our chagrin, policy and law enforcement are little influenced by science. One clear example is the federal sentence guidelines for smoked ('crack') versus intranasal cocaine ${ }^{10}$. The current dissociation between evidence and policy suggests that, far from driving discriminatory policies, integrating the neuroscientific evidence could lessen discrimination. Thus, while we applaud efforts to highlight the injustices suffered by those who use drugs, we disagree with Hart's key assertions. Discounting neuroscientific evidence does not remedy the injustices associated with drug use. These injustices must be addressed through community action, political pressure, and legal reforms, all of which stand to benefit from rigorous neuroscientific research.

Gillinder Bedi, Diana Martinez, Frances R. Levin, Sandra Comer and Margaret Haney*

Department of Psychiatry, Columbia University Medical Center and New York State Psychiatric Institute, 1051 Riverside Drive, Unit 120, New York, NY 10032, USA.

*e-mail:mh235@cumc.columbia.edu

Published online: 12 September 2017 DOI: 10.1038/s41562-017-0203-5

References

1. Hart, C. Nat. Hum. Behav. 1, 0055 (2017).

2. Diagnostic and Statistical Manual of Mental Disorders, Fifth Edition (DSM-V) (American Psychiatric Association, 2013).

3. Pavese, N. et al. Brain 126, 1127-1135 (2003).

4. Antonini, A. et al. Brain 120, 2187-2195 (1997).

5. Volkow, N. D. et al. Nature 386, 830-833 (1997).

6. Hietala, J. et al. Psychopharmacology (Berl). 116, 285-290 (1994).

7. Marek, K. et al. Neurology 57, 2089-2094 (2001)

8. Johnson, S. B., Riis, J. L. \& Noble, K. G. Pediatrics 137, e20153075 (2016).

9. Hackman, D. A., Farah, M. J. \& Meaney, M. J. Nat. Rev. Neurosci. 11, 651-659 (2010)

10. Hatsukami, D. K. \& Fischman, M. W. JAMA 276, $1580-1588$ (1996) 